\title{
ANFIS AND NEURAL NETWORK BASED FACIAL EXPRESSION RECOGNITION USING CURVELET FEATURES
}

\author{
S.P.Khandait ${ }^{\text {1), R.C.Thool }}{ }^{2)}$, P.D.Khandait ${ }^{3)}$ \\ ${ }^{1)}$ Dept. of Info.Tech., K.D.K.C.E., RSTMNU, Nagpur, India, prapti_khandait@yahoo.co.in \\ ${ }^{2)}$ Dept. of Info.Tech., SGGSIET, SRTMU, Nanded, Maharashtra, India \\ ${ }^{3)}$ Electronics Engineering Department, KDKCE, RSTMNU, Nagpur, Maharashtra, India
}

\begin{abstract}
Curvelet transform is a promising tool for multi-resolution analysis on images. This paper explains a new approach for facial expression recognition based on curvelet features extracted using curvelet transform. Curvelet transform is applied on the database images and curvelet coefficients are obtained for selected scale for image analysis. Facial curvelet features are compressed using singular value decomposition (SVD) approach. Back propagation neural network (BPNN) and Adaptive Neuro-Fuzzy Inference System (ANFIS) are used as classifiers for classifying expressions into one of the seven categories like angry, disgust, fear, happy, neutral, sad and surprise. Experimentation is carried out on JAFFE database. The experimental results show that the novel approach is a better option for extracting feature values and classifying facial expressions.
\end{abstract}

Keywords: Facial expression recognition, Curvelet transform, SVD, BPNN, ANFIS.

\section{INTRODUCTION}

The area of Human Computer Interaction (HCI) plays an important role in resolving the absences of neutral sympathy in interaction between human being and machine (computer). HCI will be much more effective and useful if computer can predict about emotional state of human being and hence mood of a person from supplied images on the basis of facial expressions and will be considered as boon for vision community. An Intelligent Biometrics systems aims at localizing and detecting human faces from supplied images so that further recognition of persons and their facial expression recognition will be easy. For classifying facial expressions into different categories, it is necessary to extract important facial features which contribute in identifying proper expressions. Recognition and classification of human facial expression by computer is an important issue to develop automatic facial expression recognition system in vision community. In recent years, much research has been done on machine recognition of human facial expressions [1-5]. In last few years, use of computers for Facial expression and emotion recognition and its related information use in HCI has gained significant research interest which in turn given rise to a number of automatic methods to recognize facial expressions in images or video [610].Multiresolution analysis techniques like wavelets are generally used in computer vision areas including facial expression recognition and face recognition [11][12][13].Many results have been achieved with wavelet transform in signal reconstruction, image analysis, facial expression recognition, etc, but wavelet transform has significant limitations in representing image edges as reported recently in [14][15]. This is due to a fact that wavelet transform can only reflect the point singularity and specialty, but difficult to express characteristics for curves and edges. In image processing, the basis of wavelet applied is isotropous, due to which it is unable to express directions of image edges accurately and sparse representation of images. Therefore, it is difficult to represent the important characteristics of facial contour and curve features using the wavelet. In order to overcome these limitations of wavelet transform, a new multi-scale analysis tool-Curvelet transform, is proposed recently [14]. Curvelet transform has good time-frequency localization characteristics, and can describe gradually to any details for an object and it can characterize the facial local information effectively.Though there are several basic results on image denoising,face recognition and palm print recognition using the curvelet features [16][17][18][19]. To best of our knowledge, there is little research on facial expression recognition with curvelet transform uptil now [20]. This paper focuses on the use of curvelet 
transform features compressed using SVD(Singular Value Decomposition) for expression recognition using ANFIS (Adaptive Neuro-Fuzzy System ) and Back propagation Neural network (BPNN) classifier and presents comparative analysis of two classifiers for curvelet-SVD features.

\section{CURVELET TRANSFORM}

The first generation of curvelet transform was proposed by Candes and Donoho [21] in 1999. In order to make the implementations simpler, faster and less redundant, the second generation of curvelet transform is proposed in 2006 [14]. It's an effective analytical method for multiresolution, band pass and directions that are considered as three important characteristics the «optimal» image representation should have from the perspective of biological point of view. Therefore, the curvelet transform has better representation capability than wavelet transform for image edges. Curvelet tiling in the frequency domain (left) and spatial domain (right) is as shown in Fig. 1. The scale and angle segmentations in curvelet transform are shown in Fig.2.There are two different digital implementations for curvelets; one is based on the USFFT (Unequispaced Fast Fourier Transform) and the other is based on wrapping idea [14]. In this paper, we select the one with USFFT for implementation simplicity and the algorithm is described as follows [14].

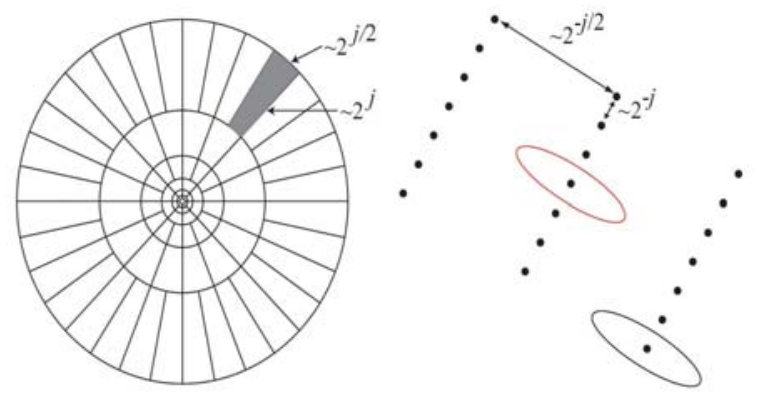

Fig.1 - Curvelet tiling in the frequency domain (left) and spatial domain (right) [14]

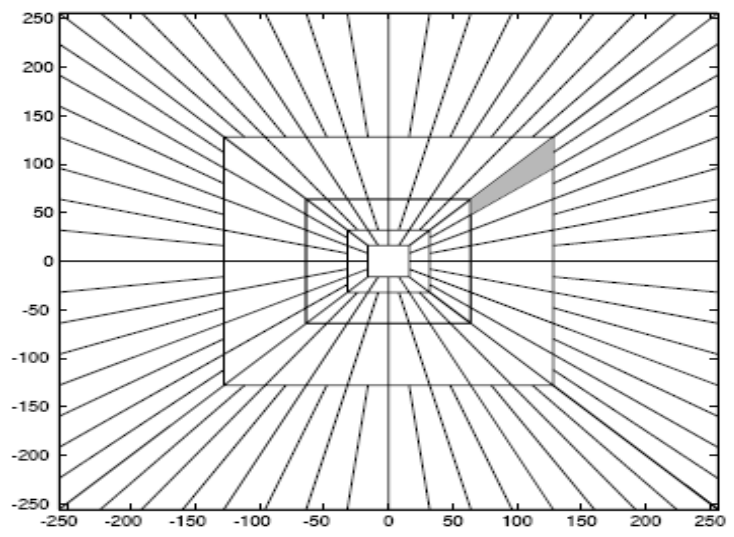

Fig. 2 - The scale and angle segmentation of Curvelet transform [14]
Suppose that we have a two dimensional discrete function

$$
f\left(t_{1}, t_{2}\right) \quad \text { with } 0 \leq t_{1}, t_{2}<n
$$

1. Apply the 2D FFT and obtain Fourier samples

$$
\begin{gathered}
f\left[n_{1}, n_{2}\right]=\sum_{t_{1} t_{2=1}}^{n-1} f\left(t_{1}, t_{2}\right) e^{-i n \pi\left(n_{1} t_{1}+n_{2} t_{2} / n\right.}, \\
-n / 2 \leq n_{1}, n_{2}<n / 2
\end{gathered}
$$

2. For each scale/angle pair $(j, b)$ resample (or interpolate) $\hat{f}\left[n_{1}, n_{2}\right]$ to obtain sampled values

$$
f\left[n_{1}, n_{2}-n_{1} \tan \theta_{1}\right], \quad \text { for }\left(n_{1}, n_{2}\right)=p_{j}
$$

where $p_{j}$ is defined in [14]

3. Multiply the interpolated object $f$ with the parabolic window $\tilde{U}_{j}\left[n_{1}, n_{2}\right]$ defined in [14] and obtain

$$
f_{I I}\left[n_{1}, n_{2}\right]=f\left[n_{1}, n_{2}-n_{1} \tan \theta_{1}\right] y_{j}\left[n_{1}, n_{2}\right]
$$

4. Apply the inverse 2D FFT to each $f_{j, l}$, hence collecting the discrete coefficients $L^{D}(j, l, k)$

\section{FACIAL FEATURE EXTRACTION}

\subsection{SINGULAR VALUE DECOMPOSITION}

$\operatorname{SVD}[22]$ is an effective algebraic feature extraction method used to compress the image features for reducing dimension. Here image is decomposed into a singular value matrix containing only a few non-zero values.

If matrix $A \in \mathbb{R}^{m \times n}$, then there exist two orthogonal matrices:

$$
\begin{gathered}
U=\left[u_{1}, u_{2}, \ldots, u_{n}\right] \in R^{m m}, \\
V=\left[v_{1}, v_{2}, \ldots, v_{n}\right] \in R^{m \times n}
\end{gathered}
$$

Which makes,

$$
A=U \sum_{,} V^{T}
$$

where,

$$
\begin{aligned}
& \sum_{\lambda}=\operatorname{diag}\left[\sigma_{1}, a_{2}, \ldots, \sigma_{p}\right], p=\min (m, n) \\
& \sigma_{1} \geq \sigma_{2} \geq \cdots \sigma_{1} \ldots \geq \sigma_{p} \geq 0, \sigma_{1}(i=1,2, \ldots, p)
\end{aligned}
$$

are all non-zero singular values of matrix $A$. They are the square root of the Eigen values of the $A A^{\text {H }}$ or 
$A^{H} A$ and

$$
\sigma_{i}=\sqrt{\lambda_{i}}
$$

\subsection{FEATURE EXTRACTION USING CURVELET AND ITS COMPRESSION USING SVD}

SVD is used along with Curvelet [23] for extracting and compressing image facial features successfully. As mentioned in [14][24], wavelets are only suitable for detecting singular points in an image and fail to represent curved discontinuities along edges. On the contrary, the curvelet transform can represent the curved changes. In image processing, the edges in an image represent significant information which can be used for representation and recognition. One significant advantage of curvelet over wavelet is that curvelet includes the detailed information on edges. Curvelet transform is successfully used for face recognition problems but very little contributions arises as for as use of curvelet transform for expression recognition is concerned. Following steps are used to extract facial features-

i) The curvelet transform is performed for each facial expression image using algorithm mentioned in section II.

ii) The coarse, detail and fine coefficients are obtained.

iii) SVD is applied to compress the coefficients.

The reconstructed images with each layer are shown in Fig. 3. From Fig.3, we can find that the detail layers represent the image sketch quite well, and include much richer information than that in the low and high frequency parts. In order to analyze the curvelet coefficients, we need a face image's expression region with resolution $n \times n$ dyadic. The images are then decomposed using the curvelet transform with scale $s$ given as -

$$
s=\log _{2}(n)-3
$$

The inner layer is a coefficient matrix corresponding to the low frequency of $32 \times 32$, and the external layer is coefficient matrix for high frequency of nxn, and the middle parts are detailed layers. For experimentation cropped face image is then resized to size $256 \times 256$ pixels. Real valued Curvelet decomposition is achieved in 5 scales. They are low frequency, first detailed layer with 64 directions, second detailed layer with 32 directions, and high frequency respectively. The size of matrix we get after curvelet decomposition is small compared to the original image size. Even then this size is still very high to use it as feature matrix therefore feature compression is achieved using SVD by using same procedure mentioned in section III. A, selecting first 32 diagonal curvelet coefficients.

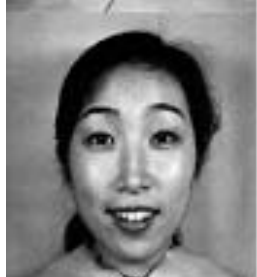

(a)

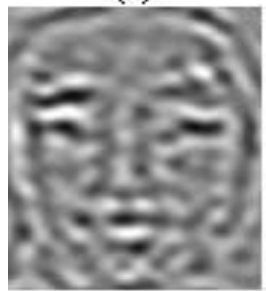

(d)

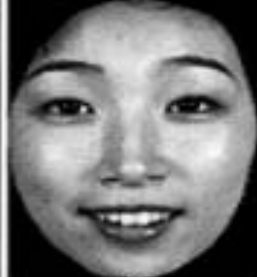

(b)

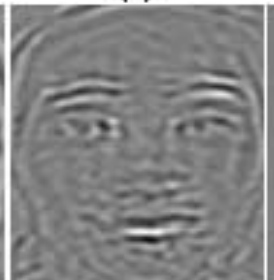

(e)

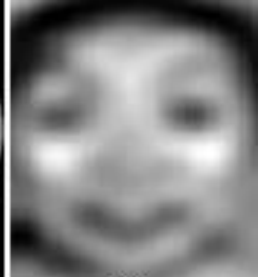

(c)

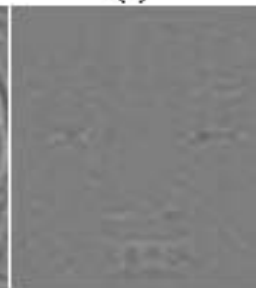

(f)
Fig. 3 - (a)-(f) original image, cropped face, low frequency, detail 1, detail 2 and high frequency parts respectively

\section{EXPRESSION CLASSIFICATION AND RECOGNITION}

\subsection{BACK PROPAGATION NEURAL NETWORK FOR EXPRESSION RECOGNITION}

The back propagation algorithm is one of the simplest and most general methods for supervised training of multilayer neural networks [25]. Fig. 4 shows the architecture of BPNN.

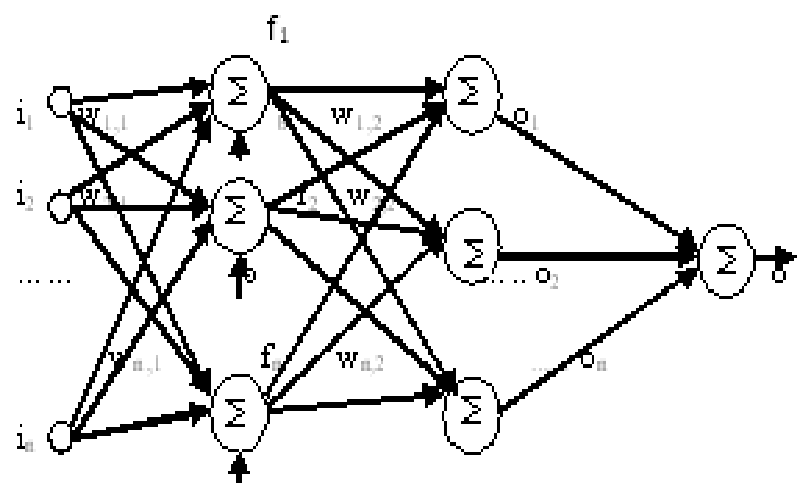

Irqut layer Hidden layerl Hidden layer2 Output layer

Fig. 4 - Architecture of BPNN

\subsection{ANFIS FOR EXPRESSION RECOGNITION}

ANFIS proposed by Jang [26] are a class of adaptive networks that are functionally equivalent to fuzzy inference systems. It represent Sugeno 
Tsukamoto fuzzy models and uses hybrid learning algorithm. Fig.5 shows architecture of ANFIS model.Thirty-two feature values obtained using curvelet-SVD method are given as an input to the ANFIS model. Initial FIS Structure is generated using grid partitioning method. Here, feature vector data is partitioned into eight groups with four inputs each. Eight ANFIS models are constructed which takes four inputs each. Three Gaussian bell membership functions are associated with each input in order to model the variation of input values (small, medium and large), so the input space is partitioned into fuzzy subspaces. In all 81 rules are generated for an ANFIS model. The premise part of a rule describes a fuzzy subspace, while the consequent part specifies the output with in this fuzzy subspace.

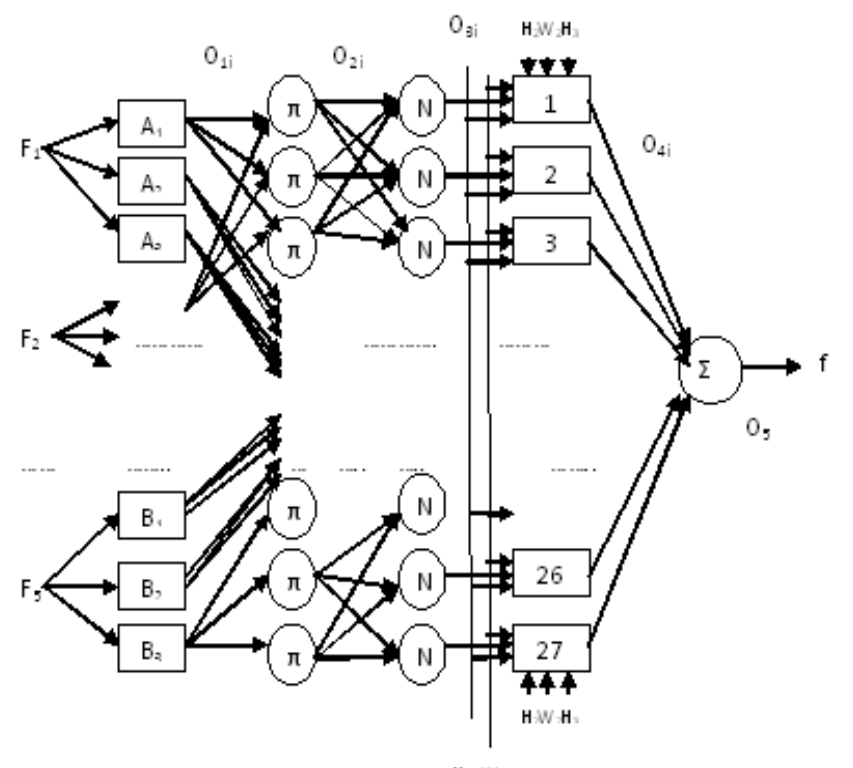

H. $W_{1}$

Layer 1 Layer 2 Layer 3 Layer $4 \quad$ Layer 5

Fig. 5 - ANFIS architecture for 4 inputs, 3 membership functions

Output of layer 5 of each model is given as input to maximum occurrence finder (Fig. 6) which finds the maximum occurrence value of particular expression. For example, output of any five models is angry, one model give neutral and remaining two models give fear expression as output then maximum occurrence finder give angry expression as output due to maximum occurrence of angry expression. Fig. 7 shows membership function before and after training ANFIS model for first four input values of SVD-Curvelet coefficients. Fig. 8 shows performance plot of Curvelet-SVD+ANFIS model. Here network learns gradually and reaches towards the goal.

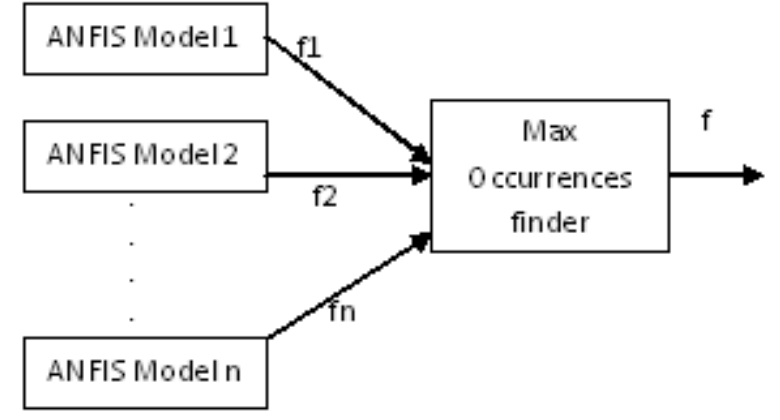

Fig. 6 - ANFIS model o/p predictor for expression recognition

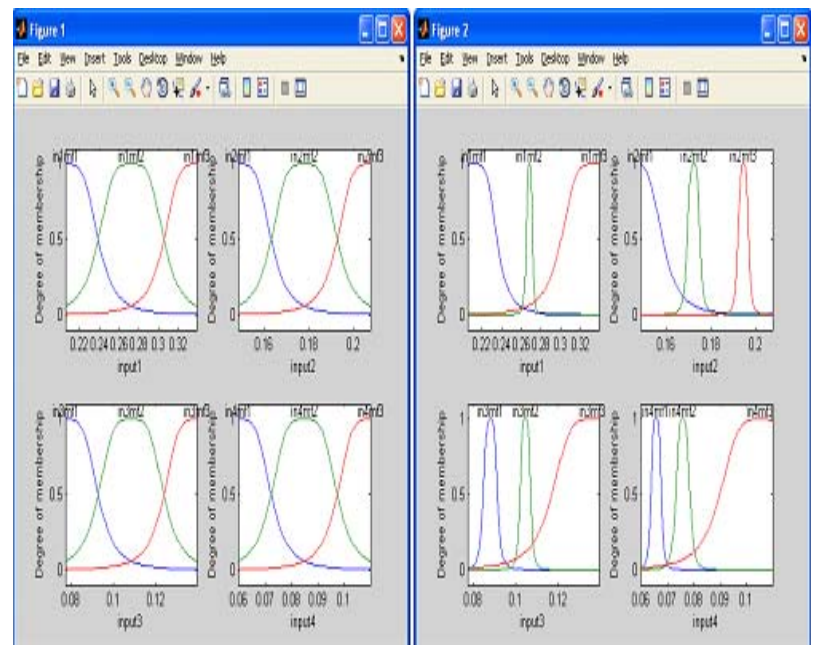

Fig. 7 - Membership function before training and after training ANFIS model

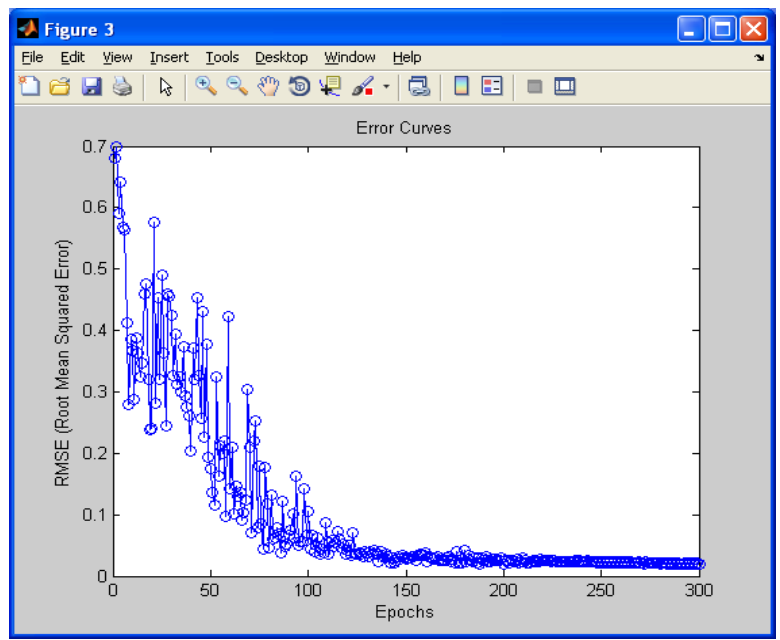

Fig. 8 - Performance plot of Curvelet-SVD+ANFIS model

\section{EXPERIMENTAL RESULTS AND ANALYSIS}

Experimentation is carried out using JAFFE database images [27], which are preprocessed using the approach mentioned in [28].Wavelet transform is applied on preprocessed images. Thirty-two 
feature values obtained using Curvelet-SVD are given to BPNN model. BPNN model with different number of neurons for two hidden layers along with variation in transfer function is tried. Neural Network Model with two hidden layers having 15 and 32 neurons each and an output layer achieved better efficiency. Transfer function used at hidden layer1 is tansig, hidden layer 2 is tansig and at output layer is linear. During training phase, BPNN model is trained for different number of samples (159, 146, 134, and 114) and achieved 100\% classification accuracy. For above experimentation selected parameters are given below - BPNN (3215-32-1), Number of epochs $=3000$, mse $=0.0001$.

During testing phase, trained model is tested for different number of samples $(30,43,55$, and 75$)$ and achieved average recognition efficiency from $93.33 \%$ to $80.00 \%$ [Table 2, Fig. 11]. Table 1 shows Confusion Matrix for testing dataset with Curvelet-SVD+BPNN based Facial Expression recognition ( 75 samples). Fig. 9 and 10 shows GUI for recognition of facial expression using CurveletSVD+BPNN and ANFIS approach respectively.

Similar to BPNN, ANFIS model is also trained and tested for same number of samples and achieved testing accuracy in the range $90.00 \%$ to 80.00\% (Table 2, Fig. 11).

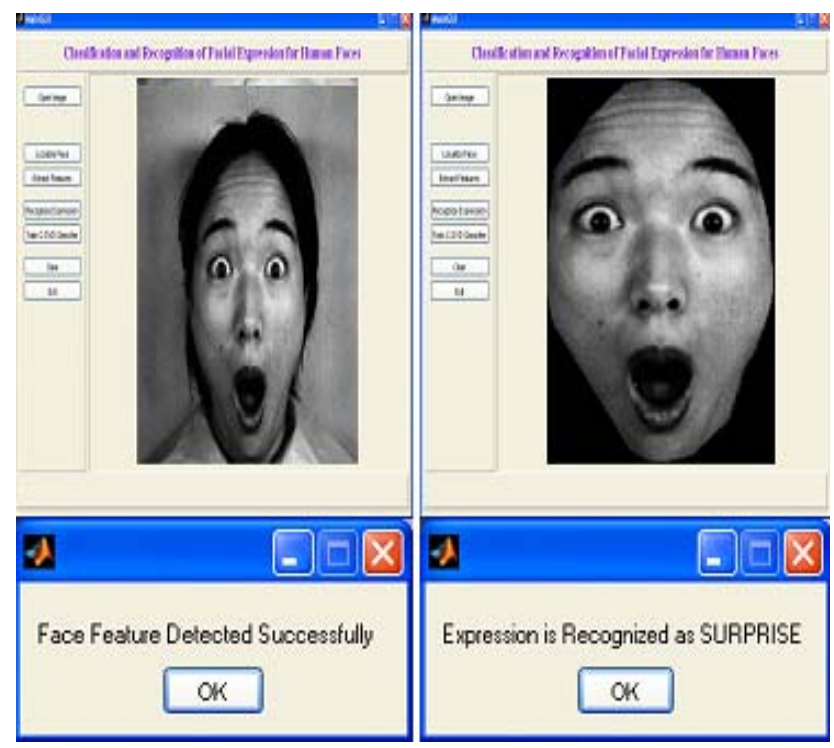

Fig.9 - GUI for recognition of facial Expression using Curvelet-SVD+BPNN approach
Classification and Recognition of Facial Expression for Human Faces

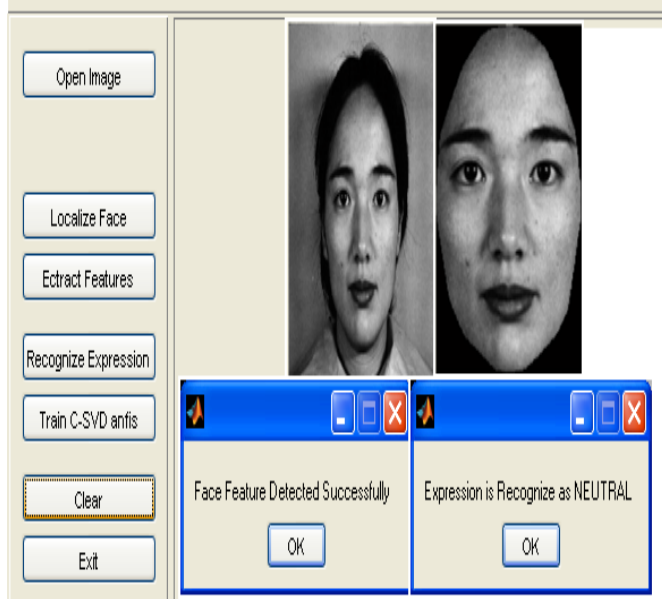

Fig. 10 - GUI for recognition of facial Expression using Curvelet-SVD+ANFIS approach

Table 1. Confusion Matrix for testset with CurveletSVD+BPNN based Facial Expression recognition (75 samples)

\begin{tabular}{|c|c|c|c|c|c|c|c|c|}
\hline $\begin{array}{c}\text { Expres- } \\
\text { sion }\end{array}$ & $\begin{array}{c}\text { An- } \\
\text { gry }\end{array}$ & $\begin{array}{c}\text { Dis- } \\
\text { gust }\end{array}$ & $\begin{array}{c}\text { Fe- } \\
\text { ar }\end{array}$ & $\begin{array}{c}\text { Hap } \\
\text { py }\end{array}$ & $\begin{array}{c}\text { Neut } \\
\text { ral }\end{array}$ & Sad & $\begin{array}{c}\text { Surp } \\
\text { rise }\end{array}$ & $\begin{array}{c}\text { Accu } \\
\text { racy } \\
\text { Rate }\end{array}$ \\
\hline Angry & 8 & 0 & 1 & 0 & 0 & 1 & 0 & 80.00 \\
\hline Disgust & 0 & 9 & 0 & 0 & 0 & 1 & 0 & 90.00 \\
\hline Fear & 0 & 1 & 8 & 0 & 0 & 1 & 0 & 80.00 \\
\hline Happy & 0 & 0 & 0 & 9 & 1 & 0 & 0 & 90.00 \\
\hline Neutral & 0 & 0 & 1 & 0 & 13 & 1 & 0 & 86.67 \\
\hline Sad & 0 & 1 & 0 & 0 & 0 & 9 & 0 & 90.00 \\
\hline Surprise & 0 & 0 & 0 & 0 & 0 & 3 & 7 & 70.00 \\
\hline \multicolumn{7}{|c|}{ Average Recognition Accuracy } \\
\hline
\end{tabular}

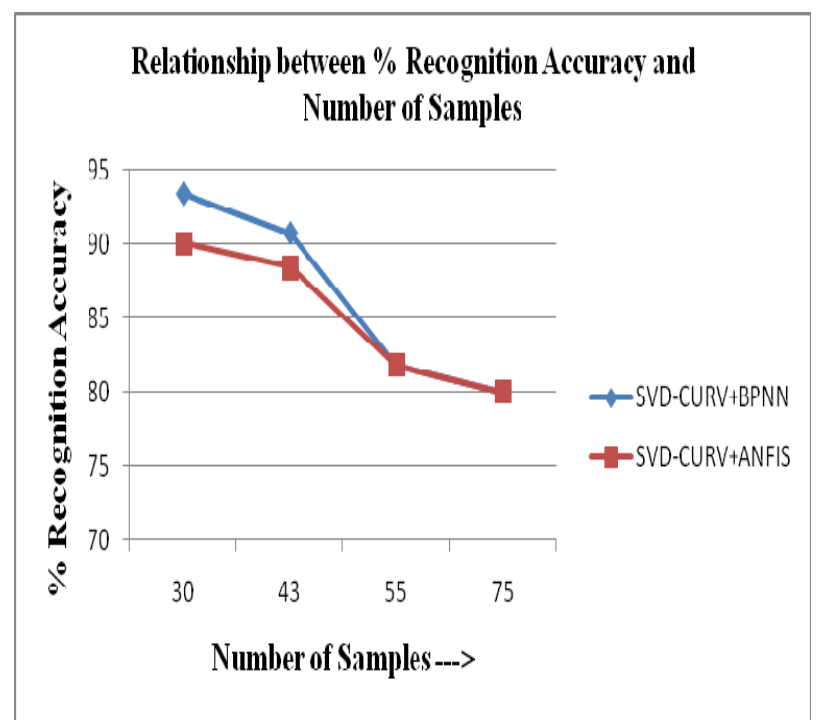

Fig. 11 - Relationship between Number of Samples and \% Recognition accuracy for Curvelet-SVD + ANFIS and Curvelet-SVD+BPNN 
Table 2. Recognition efficiency for CurveletSVD+BPNN and Curvelet-SVD+ANFIS approach

\begin{tabular}{|c|c|c|}
\hline \multirow{2}{*}{$\begin{array}{c}\text { Number } \\
\text { of } \\
\text { Samples }\end{array}$} & \multicolumn{2}{|c|}{ \% Average Recognition Efficiency } \\
\cline { 2 - 3 } & Curvelet-SVD+ANFIS & $\begin{array}{c}\text { Curvelet- } \\
\text { SVD+BPNN }\end{array}$ \\
\hline 30 & 90.00 & 93.33 \\
\hline 43 & 88.37 & 90.69 \\
\hline 55 & 81.81 & 81.81 \\
\hline 75 & 80.00 & 80.00 \\
\hline
\end{tabular}

\section{CONCLUSION}

Appearance Facial features are extracted using Curvelet transform and are compressed using SVD for efficient memory utilization. Extracted feature vector values are given as input to BPNN classifier and ANFIS classifier. Highest \% Recognition accuracy (93.33\%) was achieved using curveletSVD+BPNN approach for 30 testing samples taken from JAFFE database. Lowest \% Recognition accuracy (80.00\%) was achieved for 75 testing samples taken from JAFFE database. From Table 2 and Fig. 11 one can conclude that recognition efficiency of SVD-Curvelet+BPNN is slightly better than SVD-Curvelet+ANFIS. Thus SVD-CurveletBPNN outperforms over techniques nentioned in the literature.

\section{REFERENCES}

[1] Daw-tung lin, Facial expression classification using PCA and hierarchical radial basis function network, Journal of Information Science and Engineering, 22 (2006), pp. 10331046.

[2] Hong-Bo Deng, Lian-Wen Jin, Li-Xin Zhen, Jian-Cheng Huang, A new facial expression recognition method based on local Gabor filter bank and PCA plus LDA, International Journal of Information Technology, (11) 11 (2005).

[3] P. S. Aleksic and A. K. Katsaggelos, Automatic facial expression recognition using facial animation parameters and multi-stream HMMs, 6th European Workshop on Image Analysis for Multimedia Interactive Services, Montreux, Switzerland, 2005.

[4] Limin Ma, David Chelberg and Mehmet Clelenk, Spatio-temporal modeling of facial expressions using Gabor-wavelets and hierarchical hidden Markov models, in the Proc.of ICIP'2005, (2005), pp. 57-60.

[5] M. Pantic and Ioannis Patras, Dynamics of facial expression: recognition of facial actions and their temporal segments from face profile image sequences, IEEE transactions on Systems, Man, and Cybernetics. Part B: cybernetics, (36) 2 (2006).
[6] Ruicong Zhi, Qiuqi Ruan, A comparative study on region-based moments for facial expression recognition, in Congress on Image and Signal Processing, 2 (2008), pp. 600-604.

[7] Irene Kotsia, Ioannis Pitas, Facial expression recognition in image sequences using geometric deformation features and support vector machines, IEEE Transactions on Image Processing, (16) 1 (2007), pp. 172-187.

[8] Kakumanu P., Nikolaos, G. Bourbakis, A localglobal graph approach for facial expression recognition, ICTAI, (2006), pp. 685-692.

[9] Aleksic P. S., Aggelos K. Katsaggelos, Automatic facial expression recognition using facial animation parameters and multistream HMMs, IEEE Transactions on Information Forensics and Security, (1) 1 (2006), pp. 3-11.

[10] Spiros Ioannou, George Caridakis, Kostas Karpouzis, and Stefanos Kollias, Robust feature detection for facial expression recognition, EURASIP Journal on Image and Video Processing, (2007), Article ID 29081.

[11] Xue Weimin, Facial expression recognition based on Gabor filter and SVM, Chinese Journal of Electronics, (15) 4 (2006).

[12] Praseeda Lekshmi V., M. Sasikumar, Analysis of facial expression using Gabor and SVM, International Journal of Recent Trends in Engineering, (1) 2 (2009).

[13] Fan Chen, Kazunori Kotani, Facial Expression Recognition by SVM-based two-stage classifier on Gabor features, MVA 2007 IAPR Conference on Machine Vision Applications, (May 16-18, 2007), Tokyo, Japan.

[14] Candes E. J., Demanet L., Donoho D. L., Fast discrete curvelet transforms[R], California: California Institute of Technology, Applied and Computational Mathematics, (2006), pp.1-43.

[15] Tanaya Mandal and Q. M. Jonathan Wu, Face Recognition Using Curvelet Based PCA, 978-14244-2175-6/08/@2008 IEEE.

[16] J. L. Starck，E. J. Candes， D. L. Donoho, The curvelet transform for image denoising, IEEE Trans. on Image Processing, (11) 6 (2002), pp. 670-684.

[17] Z. Jiulong, Z. Zhiyu, H. Wei, L.Yanjun and W. Yinghui, Face recognition based on curvefaces, Proceedings of the Third International Conference on Natural Computation, Vol. 2, (2007), pp. 627-631.

[18] T. Mandal, A. Majumdar and J. Hu, Face recognition via curvelet based feature extraction, International Conference on Image Analysis and Recognition, (2007), pp. 806-817.

[19] A. Majumdar and A. Bhattacharya, Face recognition by multiresolution curvelet transform on bit quantized facial images, IEEE 
International Conference on Computational Intelligence and Multimedia Applications, (2007), pp. 209-213.

[20] Juxiang Zhou, Yunqiong Wanga, Tianwei Xu, Wanquan Liu, A novel facial expression recognition based on the curvelet features, IEEE 2010 Fourth Pacific-Rim Symposium on Image and Video Technology, (2010), pp. 8287.

[21] Candes E. J., Donoho D. L., Curvelet surprisingly effective non adaptive representation for objects with edges, curve and surface fitting, Saint-Malo, TN: Vanderbilt University Press, 1999, pp. 105-120.

[22] B. Chanda and D. Dutta Majumder, Digital Image Processing and Analysis, Prentice-Hall India, New Delhi, 2002.

[23] He Jia, Zhang Xiang Feng, Facial feature extraction and recognition based on curvelet transform and SVD, In Proc. of IEEE ICACIA09, (2009), pp.104-107.

[24] Yan Jingwen, Qu Xiaobo, Analysis and Application of Super Wavelet, National Defence Industry Press, 2008.

[25] Duda R.O., Hart P.E., Stork D.G., Pattern Classification, Second edition, Wiley student edition, 2006.

[26] J.S.R Jang, ANFIS: adaptive network based fuzzy inference system, IEEE Transactions on Systems, Man, and Cybernetics, (23), 3 (1993), pp. 665-685.

[27] JAFFE dataset, http://www.kasrl.org/jaffe.html.

[28] S. P. Khandait, R. C. Thool, P. D. Khandait, Automatic facial feature extraction and expression recognition based on neural network, (IJACSA) International Journal of Advanced Computer Science and Applications, (2) 1 (2011), pp. 113-118.

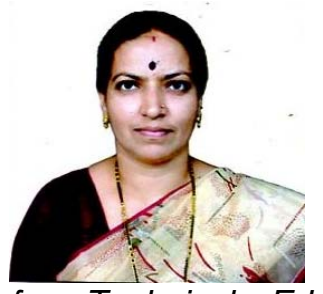

S.P. Khandait, is a research scholar and an Associate Professor in the department of Information Technology, KDKCE, RSTMNU, Nagpur, Maharashtra, INDIA. She is a Life Member of Indian Society for Technical Education (LM 23789), Associate Member of I.E.(India) (AM 81913/4), Member of I.E.T.E.,India (M 198753). Presently she is pursuing her PhD in Computer Science and Engineering. Her research research interest is Image processing, computer vision and pattern analysis.

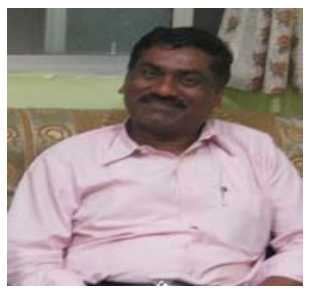

Dr. R.C. Thool is Professor in department of Information Technology, SGGSIET, SRTMU, Nanded, Maharashtra, INDIA He did his M.E. and Ph.D. from SRTMU, Nanded.

He is member of IEEE. He published more than 20 papers in national and international conferences and journals. His area of interest includes computer vision, robot vision, and image processing and pattern recognition.

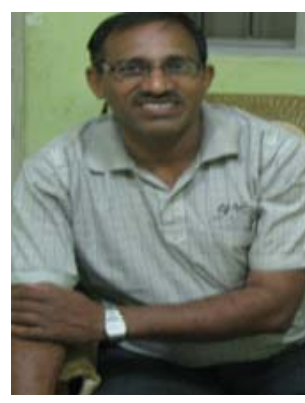

P. D. Khandait is an Associate Professor in the department of Electronics Engineering, KDKCE, RSTMNU, Nagpur, Maharashtra, INDIA. He is Life Member of Indian Society for Technical Education (LM 23790), M.I.E. (India) (M115954), M.I.E.T.E., India ( $M$ 198754). His area of research

interest includes Signal and Image processing, Biomedical Engineering, soft computing. 